\title{
Population Growth and Production of Apocyclops dengizicus (Copepoda: Cyclopoida) Fed on Different Diets
}

\begin{abstract}
This study was carried out to investigate the effects of various diets: 4 monoalgal diets: Nannochloropsis oculata (N), Isochrysis galbana (I), Chaetoceros calcitrans (C), and Tetraselmis tetrathele (T); 4 mixed algal diets: $\mathrm{N}+\mathrm{I}+\mathrm{C}+\mathrm{T}(\mathrm{NICT}), \mathrm{N}+\mathrm{I}+\mathrm{C}(\mathrm{NIC}), \mathrm{C}+\mathrm{T}(\mathrm{CT})$, and I+T(IT); and 2 nonalgal diets: baker's yeast (BY) and prepared shrimp feed (SF) on population growth and density of Apocyclops dengizicus. The type and density of diet had significant effects on the growth and density of A. dengizicus (P , 0.01). Of tested diets, $T$ and CT were optimum diets due to higher density and growth rate of A. dengizicus compared to other diets. Their high dietary value was related to the higher contents of polyunsaturated fatty acid, particularly docosahexaenoic acid (22:6n-3), eicosapentaenoic acid (20:5n-3), and arachidonic acid (20:4n-6) compared to A. dengizicus cultured on other diets. The results of the present study illustrated that T. tetrathele was the most suitable food for the culture of A. dengizicus.
\end{abstract}

Keyword: Apocyclops dengizicus 